\title{
Care pathways for glaucoma detection and monitoring in the UK
}

\author{
Robert A. Harper ${ }^{1,2}$ - Patrick J. G. Gunn ${ }^{1,2}$ Paul G. D. Spry ${ }^{3}$ Cecilia H. Fenerty ${ }^{1,4}$ - John G. Lawrenson ${ }^{5}$
}

Received: 10 September 2019 / Revised: 11 October 2019 / Accepted: 11 October 2019 / Published online: 7 November 2019

(c) The Author(s), under exclusive licence to The Royal College of Ophthalmologists 2019

\begin{abstract}
Glaucoma presents considerable challenges in providing clinically and cost-effective care pathways. While UK population screening is not seen as justifiable, arrangements for case finding have historically been considered relatively ineffective. Detection challenges include an undetected disease burden, whether from populations failing to access services or difficulties in delivering effective case-finding strategies, and a high false positive rate from referrals via traditional case finding pathways. The enhanced General Ophthalmic Service (GOS) in Scotland and locally commissioned glaucoma referral filtering services (GRFS) elsewhere have undoubtedly reduced false positive referrals, and there is emerging evidence of effectiveness of these pathways. At the same time, it is recognised that implementing GRFS does not intrinsically reduce the burden of undetected glaucoma and late presentation, and obvious challenges remain. In terms of diagnosis and monitoring, considerable growth in capacity remains essential, and non-medical health care professional (HCP) co-management and virtual clinics continue to be important solutions in offering requisite capacity. National guidelines, commissioning recommendations, and the Common Clinical Competency Framework have clarified requirements for such services, including recommendations on training and accreditation of HCPs. At the same time, the nature of consultant-delivered care and expectations on the glaucoma specialist's role has evolved alongside these developments. Despite progress in recent decades, given projected capacity requirements, further care pathways innovations appear mandated. While the timeline for implementing potential artificial intelligence innovations in streamlining care pathways is far from established, the glaucoma burden presents an expectation that such developments will need to be at the vanguard of future developments.
\end{abstract}

\section{Introduction}

The Royal College of Ophthalmologists (RCOphth) commissioned 'The Way Forward' project given an increased awareness of the growing number of patients with ophthalmic diseases of older age across the UK without a commensurate growth in the number of ophthalmologists and other human or financial resources available to treat those patients [1]. This scenario is illustrated by the comparative statistic that a decade ago there were $\sim 4.9$ million UK residents over 75 years of age, whereas by 2035 the population over 75 years is expected to be more than $80 \%$

Robert A. Harper

robert.harper@mft.nhs.uk

1 Manchester Royal Eye Hospital and Manchester Academic Health Sciences Centre, Manchester University NHS Foundation Trust Manchester, Manchester, UK

2 Division of Pharmacy and Optometry, School of Health Sciences, Faculty of Biology, Medicine and Health, University of Manchester, Manchester, UK larger at 8.9 million [2], with this growth in the elderly population precipitating increased demand for glaucoma care. The Way Forward project's modelling [1] predicts the number of people in the UK with glaucoma will increase by $22 \%$ from 2015 to 2025 and by $44 \%$ from 2015 to 2035 , with the report conceding that this growth might underestimate demand in a scenario where improved detection and management may result in more prevalent cases converting to diagnosed cases requiring ongoing management. With glaucoma management currently being responsible for an estimated 20\% of Hospital Eye Service (HES) ophthalmology out-patient workload, the previously coined term

3 Bristol Eye Hospital, University Hospitals Bristol NHS Foundation Trust, Bristol, UK

4 Division of Evolution and Genomic Sciences, School of Health Sciences, Faculty of Biology, Medicines and Health, University of Manchester, Manchester, UK

5 Division of Optometry and Visual Science, Centre for Applied Vision Research, City University of London, London, UK 
the 'bow wave of doom', a once apt metaphor for the growing demand for glaucoma care resulting from lifelong follow-up requirement in spite of low incidence, now understates what might be better considered to reflect a burgeoning tidal wave of demand. It is prescient therefore to have a review article reexamining glaucoma care pathways as part of this feature issue.

This review will focus on eye care services in care pathways relating to glaucoma detection and referral as well as diagnosis and management of glaucoma (and glaucoma related) diagnoses, and including those services falling within both primary care and secondary care settings. For the purposes of this review, models of care will be considered broadly in reference to the way health services are delivered, outlining the evidence base for care for a person, population group or patient cohort, in this case those at risk of glaucoma and those with a diagnosis of glaucoma. The care pathway, i.e. setting out the process of best practice to be followed in management of glaucoma, reflects a distillation of the best available evidence on care processes. For convenience we will consider separately the care pathways for case finding and referral filtering on the one hand, and for monitoring on the other, while recognising that the actual diagnosis of glaucoma and related monitoring services may take place in a variety of clinic environments. In setting out this review, it also needs to be borne in mind that the four countries of the UK have different structures and eye care services. With decisions on health being devolved, Northern Ireland, Scotland and Wales can make their own decisions in relation to health; however, this review will not explicitly seek to present separate analyses of the care pathways employed (indeed, arguably the countries' systems themselves are reliant on the same evidence base), rather it will highlight, where applicable, relevant similarities and differences.

Our searches broadened upon and updated an earlier published review [3] which included relevant electronic databases (MEDLINE, EMBASE, Cochrane Library, and Health Management Information Consortium (HMIC), Cumulative Index to Nursing and Allied Health Literature (CINAHL) and PsycINFO) and appropriate grey literature (e.g. websites, professional publications, and national guidelines). Keywords for bibliographic searches included the condition specific term glaucoma, profession-specific terms (optometrist, ophthalmic optician, orthoptist, nurse and ophthalmologist), and care pathway related terms (enhanced services, shared care, co-management, virtual clinic, delegated care, and referral filtering and refinement).

\section{Glaucoma detection pathways}

\section{Background}

Community optometry services for opportunistic 'case finding' for glaucoma in the UK are largely provided under the General Ophthalmic Services (GOS), with contracts being held between the NHS and optometry practice owners or contractors. Most 'sight tests' conducted in England, Northern Ireland and Wales (and all tests in Scotland) are under a GOS contract, free to the patient at the point of delivery, and from which the vast majority of referrals for suspected glaucoma have traditionally been initiated. In England, there are a number of eligible groups for NHS funded sight tests, including those with or who may be at higher risk of developing glaucoma: those aged over 60; those diagnosed with glaucoma; those aged 40 and over where a close family member (parent, sibling or child) has been diagnosed with glaucoma; and those considered at risk of glaucoma by an ophthalmologist. In Northern Ireland and Wales, NHS funded sight test eligibility from a glaucoma risk perspective is very similar, with the GOS being managed, respectively, through the Health and Social Care Board and NHS Wales. In Scotland, NHS eye tests were extended to the entire population in 2006.

\section{Glaucoma referral filtering schemes (GRFS)}

As distinct from the GOS and related arrangements for NHS sight tests commissioned nationally, in England primary eye care services (previously known as enhanced services) may be commissioned by individual Clinical Commissioning Groups (CCGs) and provide a mechanism for enhancements to an NHS sight test or private eye examination. These services include what are now termed glaucoma referral filtering schemes (GRFS), schemes which refer to: glaucoma repeat measures schemes (GRMS); glaucoma enhanced referral schemes (GERS); and glaucoma referral refinement schemes (GRRS). The NICE Quality Standard (QS180) [4] and Glaucoma Guideline update (NG81) [5], provide statements and recommendations for people planning and providing eye care services before referral such that they should consider commissioning referral filtering services for Chronic Open Angle Glaucoma (COAG) and related conditions.

In essence, GRFSs can be considered to be hierarchical in terms of the extent of accreditation and additional case finding or diagnostic testing required ahead of referral. In accordance with the NICE Glaucoma Guideline [5], the NICE Quality Standard [4] and the NICE Accredited RCOphth Commissioning Guideline [6], GRMS involves repeating intra-ocular pressure (IOP) measurement and/or visual fields, and is mostly undertaken by optometrists without additional accreditation beyond entry level, GERS adds value beyond repeat measures (e.g. dilated stereoscopic optic disc examination) and is undertaken by clinicians with the College of Optometrists' $(\mathrm{CoO})$ Professional Certificate in Glaucoma or equivalent, and finally GRRS includes tests sufficient for a diagnosis (i.e. by definition a 


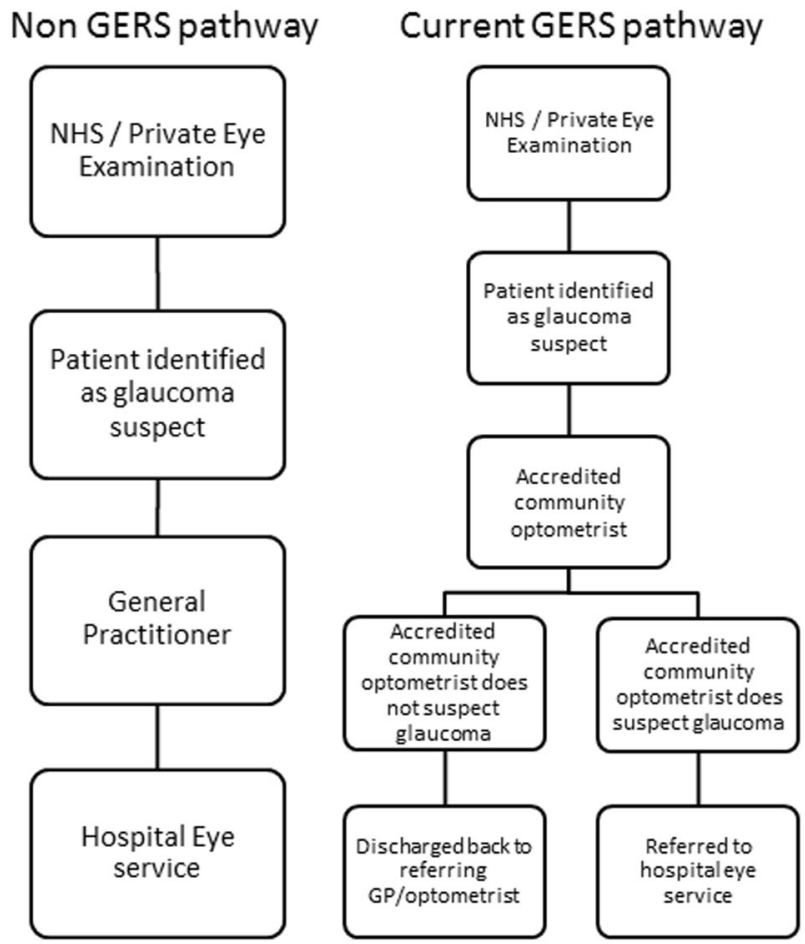

Fig. 1 GERS care pathway example from the Manchester scheme (after Gunn et al. 2018). In the traditional model referral for suspect glaucoma is via the GP. In the GERS pathway, the accredited optometrist, post examination, either makes a direct referral to Manchester Royal Eye Hospital (MREH) for specialist assessment or discharges the patient from the scheme

scheme which must also include gonioscopy) and is undertaken by those accredited to the Professional Higher Certificate in Glaucoma (previously Certificate A, or the equivalent for non-optometrists). Being relevant to not only detection but also to monitoring pathways, the matter of non-medical training and accreditation for glaucoma roles within the care pathways (including GRFS) is reviewed separately in more detail below.

An example of a published GERS pathway [7] is illustrated in Fig. 1. The Manchester scheme includes: symptoms and history evaluation; evaluation of general health, medications, previous eye history and family history; evaluation of glaucoma risk factors; visual acuities; anterior segment assessment; van Herick assessment of the angle; Goldmann applanation tonometry; pachymetry; visual field assessment; and a dilated fundus examination incorporating clinical optic disc assessment using slit-lamp biomicroscopy (with either a 78D, 60D or 66D lens).

One cautionary point to note when considering the literature on GRFS, however, is the potential for various terms to be applied in different settings and at different time points. For example, true GRRSs appear to be few and far between (i.e. in the strict sense of the need for such services to include testing sufficient for a diagnosis of glaucoma and to be delivered by appropriately accredited practitioners in accordance with NICE); however, it is clear that the 'referral refinement' term was used historically for many years to describe some GRFSs before NICE guidance was published, for example the original Manchester scheme [8]. Furthermore, the term referral refinement is still used to describe some GRFSs in other countries, for example, the Carmarthenshire GRRS, a scheme without inclusion of gonioscopy [9], or in a jurisdiction where NICE does not apply, for example, the 'referral refinement' scheme described by Barrett et al. [10] in Ireland.

GRFS have been in operation in the UK for almost two decades and Manchester's GRRS, now termed the Manchester GERS to fit with the NICE definition, was the first such scheme to be established [8]. The Way Forward report [1] highlighted the national need for referral filtering and indicated that GRFS are widespread, with 66\% (31/47) of glaucoma leads interviewed indicating referral filtering is in operation in their locality. The GRMS category of referral filtering appears most widespread in England, with data from the Local Optical Support Unit (LOCSU) listing $\sim 60$ such schemes across Local Optical Committees in England [11], in contrast to $\sim 15$ "glaucoma referral refinement" schemes in self-evidently far fewer regions, and where strictly speaking, as implied above, many of which are likely to be representative of the GERS model versus true GRRS as the term has now come to be defined. From the stakeholder perspective, patients and others including commissioners appear to respond positively to these community services $[12,13]$.

\section{Effectiveness of traditional case finding and GRFS}

Community optometrists identify the vast majority of suspect glaucoma and ocular hypertension (OHT) cases in the UK through GOS sight testing [14-17]; however, without referral filtering (which at least in part works well through 'enriching' the target population of interest [18]) the false positive rate is considered to be high. This latter finding appears to be so Europe-wide, with a recent study concluding that the accuracy of referrals is poor in the UK and other countries, and that a combination of criteria and raising the IOP threshold for IOP-only referrals are needed to cut waste in clinical care [19]. Early UK studies of referral for glaucoma by optometrists illustrated optometrists" criteria for using "screening" tests and their subsequent decisions on the referral of suspects varied widely [20-22]. Consequently, the false positive rate from the traditional detection pathway through case finding has typically been found to be high, for example, ranging from 20-65\% [20, 21, 23-27]. Vernon and Gosh [28] established that the provision of specific referral guidelines, circulated to all optometrists working within the catchment area, had 
little effect upon false referrals. It was against this background that referral refinement was introduced and an early evaluation of this first GRFS, the Manchester GRRS, observed a reduction in the FP rate from $\sim 40 \%$ to $~ 10 \%$ [8]. Subsequently, Ratnarajan et al. [29] published the first multisite review of GRFS in the UK, using a timeline encompassing all the major changes in clinical guidelines and practice following the publication of the original NICE guideline (CG85) [30] and including a sample of over 1000 patient outcomes in a retrospective observational time series study. Their findings showed a significant impact on the false positive rate, with the first-visit discharge rate for optometrists with a specialist interest (i.e. GRFS participants) being significantly lower at $14.1 \%$ compared with $36.1 \%$ for those optometrists not GRFS accredited [29]. There are fewer studies evaluating the false negative rate within community GRFS. One study in Carmathanshire [9] included a retrospective analysis of 100 sets of notes and optic nerve images of patients not referred through the scheme. The authors quoted a FN rate of $3-10 \%$ and concluded their findings indicated 'no compromise on patient safety' [9]. Ratnarajan et al. [31] included consultant clinical review of non-referred patients, albeit on only a limited sample of 34 discharged subjects willing to attend the hospital for review, and concluded that the false negative rate of the optometrists in their scheme was $15 \%$, although no cases of glaucoma were missed. More recently, the largest study of the false negative rate of a GRFS was published using a methodology where a proportion of nonreferred study patients were all examined in the same NICE aligned clinic model as that for usual care referred patients [7]. The findings showed a low false negative rate, no clinical incidents associated with false negatives, and concluded that this GERS model was an effective GRFS.

\section{Effectiveness of the enhanced GOS model}

Outside of the locally commissioned arrangements for GRFSs in England, the enhanced GOS in Scotland also appears to offer an improvement over traditional case finding arrangements. For example, in an early evaluation following the introduction of GOS changes in Scotland in 2006, Ang et al. [32] demonstrated an improvement in the quality of glaucoma referrals from community optometrists in northeast Scotland, with a corresponding reduction in false positive referrals. El-Assal et al. [33] retrospectively reviewed hospital glaucoma clinic data from 1622 patients in two 6 year periods, one before the introduction of the new GOS contract, between 2000 and 2006, and the other after the contract was introduced, between 2007 and 2012. They reported that patients were now being referred earlier, with shorter waiting times for hospital appointments, and with referrals comprising more glaucomatous cases and fewer false positives. Since then, SIGN 144 (Glaucoma referral and safe discharge) [34] has been published and this too appears to have afforded a further improvement in referral accuracy. In a retrospective study, Sii et al. [35] observed a significant decline in the first visit discharge rate when comparing two 2-month periods both before (29.2\% first visit discharge rate) and after (19.2\% first visit discharge rate) the publication of SIGN 144, while also observing room for improved adherence to referral criteria. On a more specific clinical query, Annoh et al. [36] recently examined the accuracy of referrals for primary angle closure related diagnoses in Scotland. In 769 consecutive referrals they determined that community optometrists had 'good' ability to detect eyes at risk of angle closure, with only $12 \%$ of those referred for possible angle closure being discharged at the first visit. In Wales, the GRFS established in Carmarthenshire has been determined to be clinically effective, with a 53\% reduction in referrals to the HES [9]. In Northern Ireland, Black et al. [37] describe an audit comparing optometrists' practice in assessing signs for glaucoma pre- and post-training and accreditation for working within a GRMS. Participation in the scheme had a positive impact on practitioners' choice of pre-referral assessments performed in primary care.

\section{Cost-effectiveness of GRFS}

In comparison to data on clinical effectiveness, there remains a paucity of data on the cost-effectiveness of GRFS. In their realist review, Baker et al. [3] noted that cost savings from GRFS for suspected glaucoma are based on the number of HES referrals prevented versus the schemes' costs. Using community optometrists with a specialist interest in glaucoma to filter referrals from other optometrists varies in cost-effectiveness from cost-neutral [38] to producing a small $[8,39]$ or substantial $[9,40]$ saving compared with equivalent HES care. Cost-effectiveness appears to depend upon scheme activity and assumptions in the financial model. Henson's early evaluation of 'referral refinement' [8] certainly sparked reasonable questioning of such assumptions [41, 42]. Interestingly, the introduction of a GRMS in South London (i.e. where the original referring community optometrists repeated tests to confirm abnormality prior to referral or non-referral to the HES) produced a $62 \%$ cost-saving compared with the usual HES tariff [38]. Henson et al. [8], Devarajan et al. [9] and Parkins and Edgar [38] all based their financial analysis of GRFS on the assumption that more than one HES visit is required prior to discharge for false positive referrals. Henson et al. [8] based their analysis on the assumption that false positive patients who would have attended the HES without a GRFS would have had on average 2.3 clinic visits before being discharged, a figure that is based on a review of new referrals 
to the MREH made before the introduction of the Manchester scheme. Devarajan et al. [9] also based their financial analysis on the assumption that a false positive referral would otherwise have an average of 2.3 clinic visits before discharge and Parkins and Edgar [38] and Ratnarajan et al. $[29,39]$ assumed an average of 2.1 visits prior to discharge. A more recent study analysing the costs of the Manchester GERS [43] indicates that $\sim 2.2$ visits need to be avoided to make their scheme cost saving, albeit the authors also note that reducing the volume of referrals will reduce waiting time for an outpatient appointment and therefore GERS has the potential to reduce waiting times as well as false positive referrals. One interesting innovation in the past decade is the use of a virtual clinic model (more typically used in monitoring schemes and discussed below) to refine [40] and triage [44] community referrals, with the former study in Portsmouth showing $11 \%$ of patients 'attending' virtual clinics being accepted into the HES, while releasing 1400 clinic slots per year to the local NHS Trust. A further potential GRFS innovation is the use of technology. For example, the GATE study [45] compared optic nerve and nerve fibre layer imaging technologies for filtering referrals alongside VA and IOP data, and while showing cost effectiveness, the filtering resulted in one in seven cases of glaucoma being inappropriately discharged; however, some form of automated referral filtering seems likely to be realised in the future as technology advances.

\section{Care pathways for glaucoma diagnosis and monitoring}

\section{The development of 'shared care'}

Shared care or co-management has come to represent the sharing of clinical management responsibilities between two or more health care professionals (HCP) from different disciplines, although it is clear the term 'shared care' may mean different things to different people, for example: data collection only, data collection and decision making by protocol, and data collection and autonomous decision making. Shared care may also include virtual clinic activity (see below). In an early editorial on the topic of shared care, Hitchings [46] welcomed the concept, while highlighting legitimate concerns about how such schemes might operate, particularly outside the confines of the hospital, and in an era pre-dating the evidence base now held on the effectiveness of non-medical HCP working in glaucoma care. The timeline chart in Fig. 2 illustrates factors that have promoted the development of non-medical HCP's engagement in glaucoma shared care in the past 30 years.

A decade ago Vernon and Adair [47] set out to determine the number and nature of shared care schemes for glaucoma and glaucoma suspects operating in England. Their survey showed that even before the outcome of the then Department of Health shared care pilots had been published, $\sim 50 \%$ of ophthalmic departments were running schemes for glaucoma; however, these authors concluded at the time that most schemes contributed only modestly to the overall volume of glaucoma care, with the majority of glaucomarelated consultations being undertaken by ophthalmologists. This scenario has almost certainly changed in recent years. The Way Forward report [1] noted $88 \%$ of glaucoma clinical leads interviewed had incorporated non ophthalmologists into expanded roles. Harper et al. [48] published a comprehensive evaluation of the scope of practice of optometrists working in the UK HES. Their results, with an excellent response rate of 70 of 79 survey invitations, described the substantial majority of respondents $(96 \%)$ undertook "extended" clinical roles, with glaucoma being the leading extended role service provided by optometrists (92\% of respondents providing extended role services). In terms of the general scope of practice, this survey found evidence that optometrists engaged in these extended roles did so with a significant degree of autonomy, with only $23 \%$ of clinics being reported to 'never go ahead' without an ophthalmologist also being present. While the presence or absence of a consultant or other member of the medical team within the clinic does not necessarily mean that medical clinical decision support was unavailable, across all extended role services in general, less than a third of respondents replied that they 'often' or 'always' required consultation with medical colleagues, a response that was provided within the context of a senior optometrist experienced at working within the extended role, and not an optometrist in training for the role. In Vernon and Adair's survey [47], the shared care staff members were able to prescribe medication for glaucoma in only eight schemes (12\%). As will be evident from developments summarised in the timeline in Fig. 2, there is now a much changed scenario, not least in terms of the prescribing of therapeutics.

In terms of effectiveness data, the first high quality evidence for the potential role of non-medical HCPs in glaucoma monitoring was derived from the Bristol shared care glaucoma study. This randomised control trial (RCT) compared community optometry monitoring of stable glaucoma to routine care in the hospital glaucoma clinic, and determined that optometrists could take clinical measurements of comparable quality to usual care $[49,50]$ and that over the 2-year study period, there was no difference in patient outcomes between the two arms [51]. Since this $\mathrm{RCT}$, there is further supportive evidence that specialist optometrists, when additionally trained and accredited in glaucoma, can make appropriate diagnostic and clinical management decisions compared with a subspecialist 


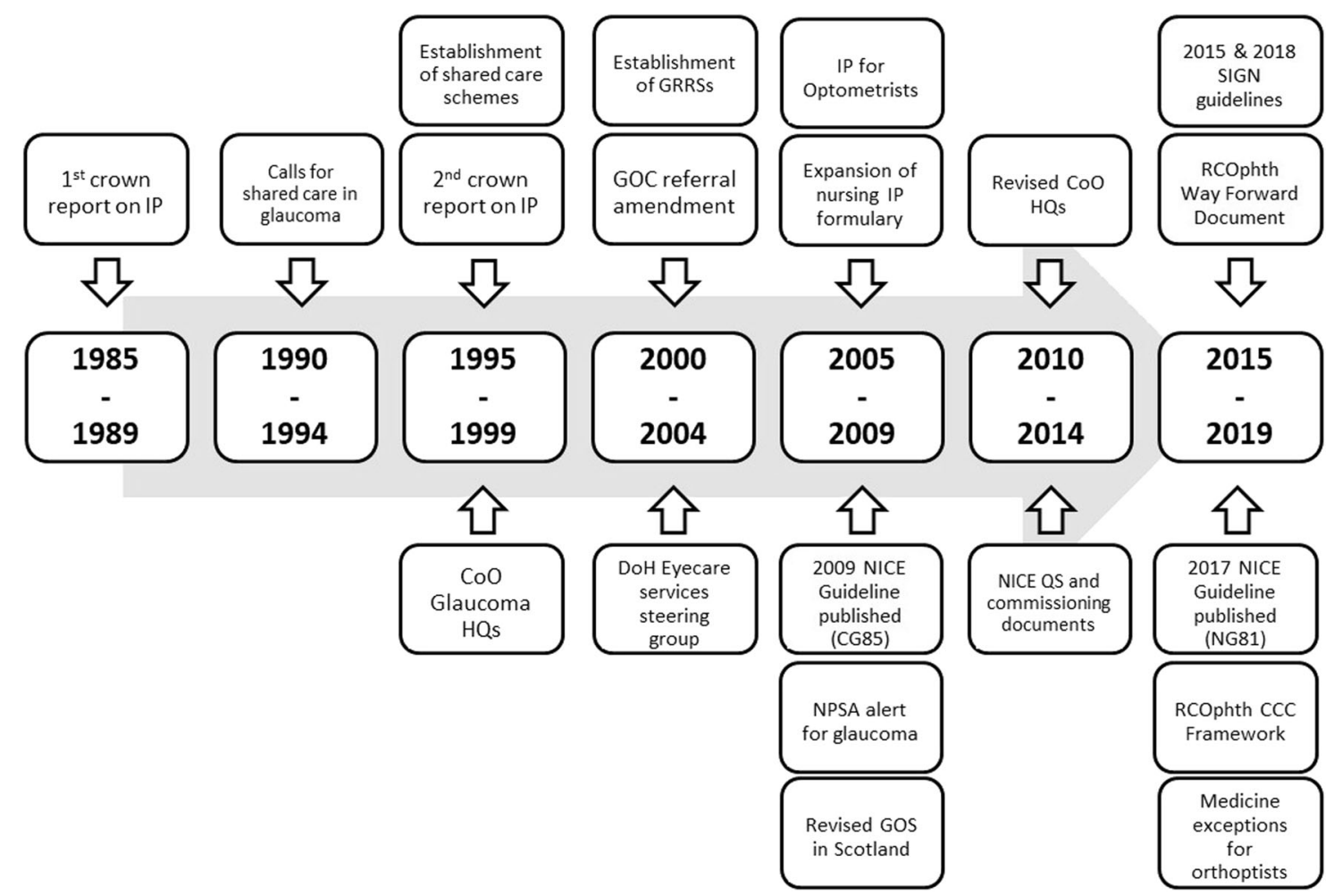

Fig. 2 Timeline chart incorporating key legislation, guidelines, safety alerts and key professional developments in the three decades between 1989 and 2019. The figure shows the wider context and enablers for progression of non-medical HCP's engagement in glaucoma care pathways beyond those traditionally encountered in previous decades. Key to acronyms: IP (independent prescribing); $\mathrm{CoO}$ (College of

ophthalmologist reference standard [52-56]. In terms of community based co-management, other observational studies have concluded that with further glaucoma training, optometrists can be an acceptable alternative to hospital care for selected glaucoma patients and those at increased risk of glaucoma [57-59].

The literature on nursing roles in the glaucoma care pathway provides evidence of roles in patient education, including improving adherence, and in helping patients with sight loss, for example, Ney [60]. It is also clear that nurseled glaucoma clinics have been in existence for many years, for example Johnson et al. [61], including a role in glaucoma related laser procedures [62]; however, the authors are unaware of empirical studies evaluating nursing decision making performance in glaucoma versus, for example, studies reporting on the taking of specific clinical measurements (e.g. tonometry [63]). Similarly, in the UK there does not yet appear to be orthoptic specific evidence for performance in glaucoma care roles, albeit it can be legitimately argued that the overall HCP evidence base affords evidence well beyond "proof of concept", and as such the evidence available is likely to be applicable to any professional with appropriate training and accreditation and
Optometrists); GRRS (Glaucoma Referral Refinement Scheme); GOC (General Optical Council); DoH (Department of Health); NICE (National Institute for Health Care and Excellence); NPSA (National Patient Safety Agency); GOS (General Ophthalmic Service); HQ (Higher Qualification); SIGN (Scottish Intercollegiate Guidelines Network); and CCC (Common Clinical Competency)

experience (a matter considered in more detail below). Indeed, while conceding that nursing roles in the wider UK health care setting were inherently less independent than those of optometrists, The Way Forward report [1] proposed that the Ophthalmic Common Clinical Competency Framework (OCCCF) [64], discussed in context below, should help to ensure that a consistent level is achieved by all HCPs, regardless of professional grouping or starting point.

\section{Risk stratification in the glaucoma monitoring care pathway}

It is widely recognised that there is significant diversity of case complexity in glaucoma and glaucoma-related diagnoses, with potential vision related outcomes varying from minimal lifetime risk of sight loss through to significantly higher risk of either imminent or longer term sightthreatening glaucoma. This range in case complexity is reflected in commensurate variations in the care pathway required for glaucoma patients, and in the level of training and skills required by participating non-medical HCPs, now significantly to the fore in dealing with the burden of glaucoma. The RCOphth and the $\mathrm{CoO}$ have recently 
System and Assurance Framework for Eye-health (SAFE) - GLAUCOMA SERVICE SYSTEM

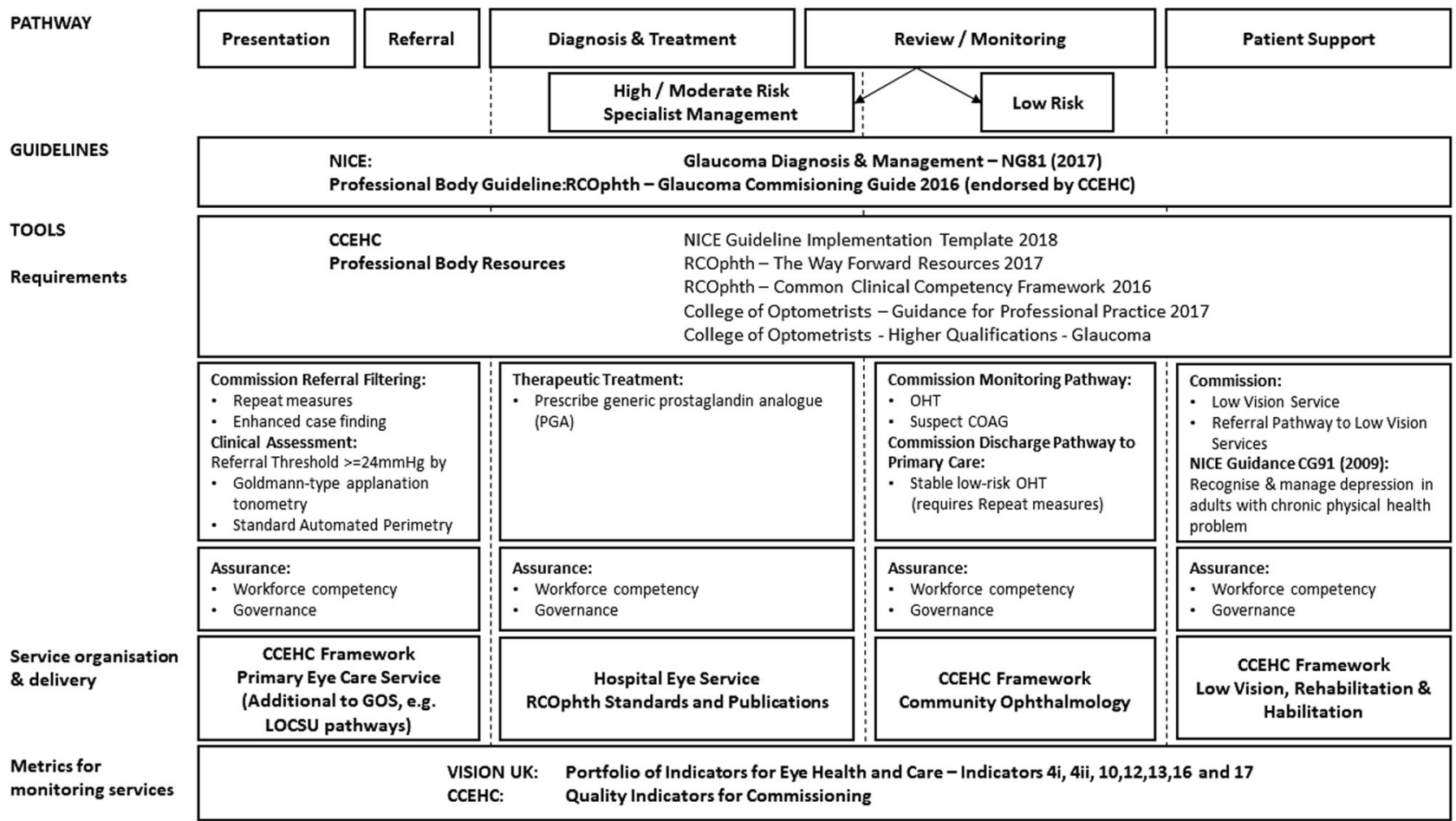

Fig. 3 Clinical Council for Eye Health Commissioning (CCEHC) System and Assurance Framework for Eye-health (SAFE) Glaucoma Service System

produced joint ophthalmic services guidance on primary eye care services, community ophthalmology and general ophthalmology [65] and The Way Forward summary for glaucoma [1] describes stratification of patients' risk of sight loss being used to organise review at virtual clinics, HCP specific clinics and consultant clinics, while also noting the importance of clarity for discharge policies for those referred with possible glaucoma (and found not to have it) or for OHT, and including the need for clear instructions for re-referral.

Recent years have witnessed improvements in eye care services within the four home nations of the UK, with each country developing individualised pathways for delivery of eye care in line with government and local health service policies [66], with public health promotion, improvement in provision and accessibility, and the use of a multidisciplinary model being seen as key [5, 6, 34, 67, 68]. In England, the Clinical Council for Eye Health Commissioning has produced a system assurance framework for eye health, SAFE, (see Fig. 3), encouraging and supporting services in primary and secondary care to develop pathways involving suitably qualified HCPs to help with the increasing capacity and demand pressures [69]. Of note here is an important reminder of the significance of the need for wider patient support elements within the pathway, including for example, the potential need for referral for low vision rehabilitation, for completion of the Certificate of Visual Impairment facilitating access to wider support and services, and for considering the risks of depression.

From the perspective of the community setting, LOCSU [11] in England has produced a glaucoma monitoring pathway for those patients considered suitable for community monitoring (see Fig. 4).

\section{Virtual clinics in glaucoma care}

Caring for people with glaucoma and related conditions is an enormous task requiring multi-professional collaboration, discussed above, as well as innovations in service delivery. One such innovation is the 'virtual' clinic. In these models of care, some of which utilise electronic patient records (including web-based data recording), patient data is usually collected by technicians, ophthalmic nurses and/or non-specialist orthoptists or optometrists, with subsequent 'virtual' consultant ophthalmologist/expert clinician data review and decision making [70, 71]. The care model is usually implemented for follow up attendances either in hospital or community clinic settings (including services that make use of a mobile clinic facility). These clinics are intended to: maximise appointment capacity and reduce waiting times; provide a 'one stop shop' with all tests being performed on the day; and ensure that people who can be 
Fig. 4 The Local Optical Support Unit (LOCSU) has developed an integrated service pathway made available to local optical committees and commissioners to consider as a tool for developing glaucoma care pathways for monitoring patients with glaucoma and related conditions in the community

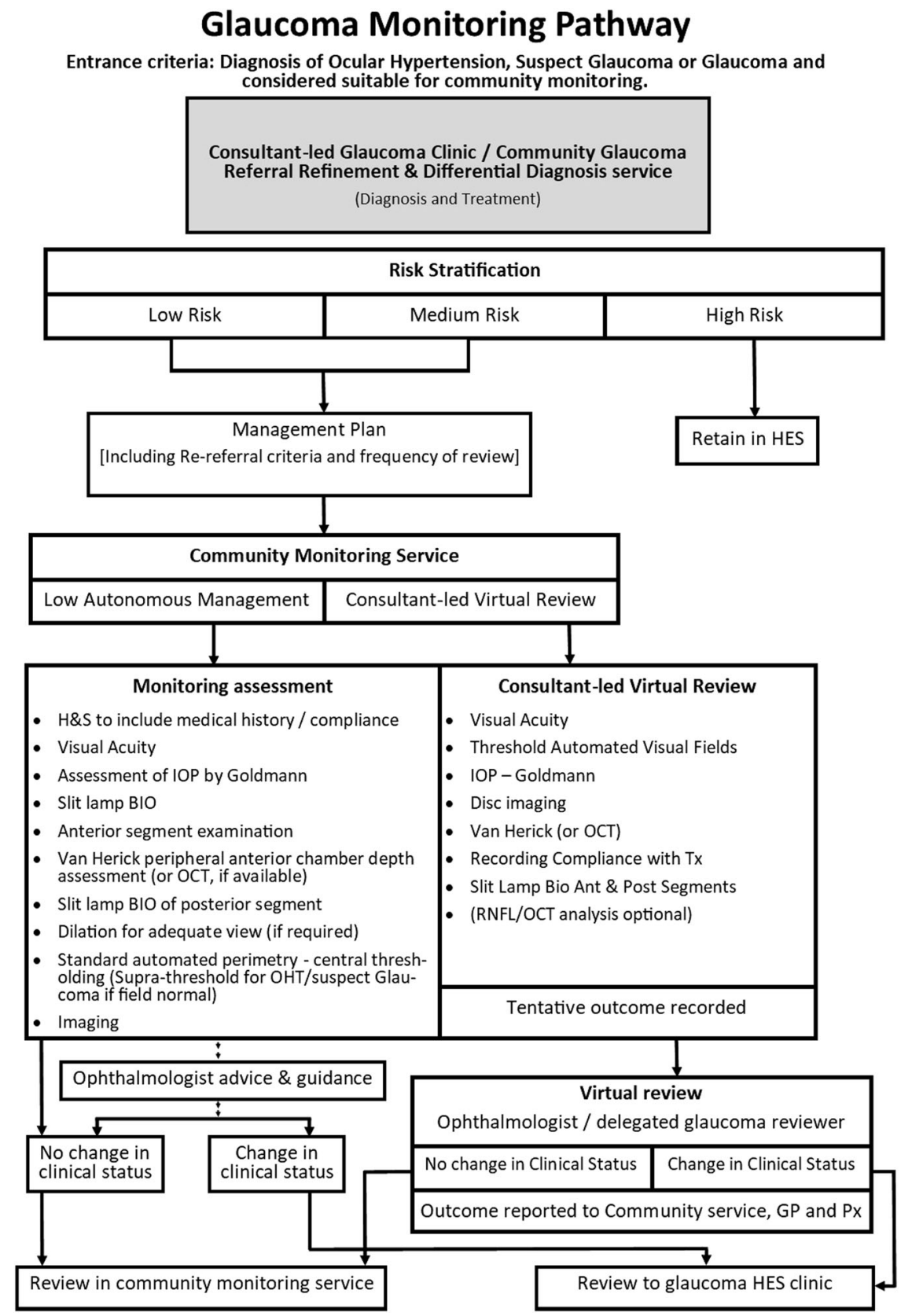

discharged are discharged sooner by consultant or expert clinician review. Alternatively, and as noted above, the virtual model has been used to refine community referrals [40].

A recent survey by Gunn et al. [72] determined that glaucoma virtual clinics are employed by a large proportion of HES units, with many others seeking to develop such services. Clinical leads largely rate efficiency, patient safety and the perception of patients' acceptability to be at least equivalent to standard care. The RCOphth Standards for Virtual Clinics in Glaucoma Care [73] aimed to define minimum standards for the development and implementation of virtual clinics for glaucoma in the secondary care setting and was produced by expert panel consensus. The document provides recommendations on patient suitability for virtual clinic monitoring, test procedures and processes, staffing, data collection and governance. The panel recommended that patients with OHT, suspected open angle glaucoma, or early or moderate glaucoma (open angle or pseudophakic patients with a history of angle closure) in the worse eye may be suitable for this type of clinic model. Gunn et al.'s survey findings suggest that most respondents were already working within these recommendations, with $90.5 \%$ using virtual clinics to assess patients with OHT and $71.4 \%$ assessing glaucoma suspects [72]. However, this survey did note that $28.6 \%$ of respondents included patients 
at any stage of disease, provided their condition was deemed to be 'stable'. The 'Standards' do allow for Consultant discretion in deciding on whom to refer to a virtual clinic, although they do recommend excluding patients who have non-glaucomatous pathology. In terms of safety, a study by Clarke et al. [74] showed that virtual clinics can be safe, with misclassification events at just 1.9\%. Furthermore, Kotecha et al. [75] and Court and Austin's [76] studies have shown that patients are accepting of virtual clinics under certain provisos, albeit these studies involved patients from a mainly Caucasian population with low risk eye disease and arguably further qualitative research is necessary to understand more about both different patient groups and clinicians' experiences and perceptions of virtual clinics, to further inform approaches to this model of care. The Way Forward report highlighted familiar barriers to use of virtual clinics, raising clinicians' concerns surrounding taking away the human, face-to-face element of decisionmaking [1], although Gunn et al.'s survey [72] indicates that commonly reported barriers for clinicians relate to practical issues including having insufficient time, funding, staffing or space resources to establish and run virtual clinics, with clinical leads working in acute trusts or major teaching hospitals being more likely to have overcome these barriers.

While not the same as a virtual clinic, the reviewing of cases awaiting future appointments is another option for evaluating the clinic backlog. For example, in a recent study aptly titled 'tackling the NHS glaucoma clinic backlog issue' Broadway and Tibbenham [77] describe examining 9290 cases in a 3 year study evaluating whether previously planned clinic reviews were appropriate. They concluded that departments experiencing significant backlog issues should consider using trained glaucoma sub-specialist consultants to review the planned follow up management of patients within a backlog deficit. Other units have considered external providers to help with backlog issues, including the use of providers employing a virtual clinic model [70]. The Way Forward report highlighted some advantages of such an approach while cautioning on the need for careful retention of patient data collected by outside providers in order to avoid clinical governance risks associated with loss of data and the potential adverse consequences to patient care [1].

\section{Consultant-delivered clinics}

Consultant-delivered clinics represent the conventional model of glaucoma care, with patients attending an ophthalmic outpatient clinic traditionally staffed by a team of junior and middle grade ophthalmologists led by a consultant. From a historical perspective, nurses would perform visual acuity assessment and ophthalmic technicians would have performed visual field testing and optic disc imaging.
With the increasing demand upon glaucoma services outstripping capacity, newer models of service delivery have been developed as described above. Nevertheless the conventional consultant delivered clinic continues to provide a vital role in glaucoma care:

- Patients still require diagnoses to be consultant-led, and this requirement becomes particularly important for secondary glaucomas or other conditions which may mimic glaucomatous signs and symptoms, including other ocular or neurological disease.

- Once glaucoma is diagnosed and treated, patients who do not respond to (or who cannot tolerate) first and second-line therapies require consideration of surgery or other interventions requiring consultant leadership.

- Some patients have complex or multiple ocular comorbidities which do not easily fit into the more protocol-driven shared care or virtual clinic models and require face-to-face consultations.

- Patients who have undergone glaucoma surgery need consultant-led review in the immediate post-operative period to ensure surgical success.

Hence the case mix of consultant-delivered clinics has evolved from delivering care to all patients with glaucoma related conditions, to one which is predominantly reserved for moderate to high risk glaucoma cases.

Personnel resourcing consultant-delivered clinics have also evolved over time. The development of shared care and virtual clinics and the extended clinical skills acquired by HCPs provides the opportunity for optometrists, nurses and orthoptists to become integrated into consultant-delivered clinics, fulfilling the same roles as junior and middle-grade ophthalmologists. The Way Forward report [1] highlighted that this direct consultant supervision of HCPs provides a solution to meet capacity requirements, as well as providing an opportunity for teaching, training, and professional development of non-medical HCPs, a factor contributing to job satisfaction and retention of HCPs, and ensuring facilitation of increased autonomy in shared care clinics for lower risk cases without direct consultant supervision.

\section{Evolution of the consultant role within the care pathway}

In the same way that the roles of non-medical HCPs have been extended to delivering glaucoma services [47, 48], the role of the consultant ophthalmologist has also evolved alongside the adoption of newer ways of working. Clinical assessment and management of individual patients remains a key role, although increasingly this role is confined to higher risk and more complex cases. Performing surgery and other interventional procedures also remains largely in 
the domain of ophthalmologists and led by consultants. Additionally consultants have always led the teaching and training of ophthalmologists and other HCPs. With the further development and expansion of the shared care and virtual clinic models, the demand of being a lead-trainer for HCPs has increased, whilst also retaining responsibilities for the teaching and training junior ophthalmologists. Furthermore, the role of 'Clinical Lead' for glaucoma services now demands not only the skills of the consultant to lead on the re-design of service models and the training and accreditation of HCPs, in addition to their own clinical responsibilities of outpatient and surgical sessions, but they also need to fulfil a governance role in oversight of these services and responsibility for the safety and effectiveness of these models of care. Whilst those HCPs with recognised higher qualifications may take full responsibility for the care they deliver, many HCPs fulfil their roles under supervision, directly or indirectly, by a consultant. The absolute numbers of patients who are registered to a named consultant may therefore steadily increase as an ophthalmic unit develops and expands newer models of care to meet increasing demand. Consultant ophthalmologists have always taken ultimate responsibility for patients seen and managed by 'other members of the team', however this team has moved from the confines of consultant-delivered clinics, to separate HCP clinics, sometimes performed at times and locations remote from the consultants' own clinics. Overseeing quality of care may become increasingly difficult. Furthermore, as many of these newer models of care are protocol driven and directed specifically to the detection and monitoring of only glaucoma conditions, the risk of other diagnoses going undetected increases. These potentially undiagnosed conditions may range from cases of mild dry eye or developing cataract, to unrecognised systemic side effects from glaucoma therapies, to other more serious non-glaucomatous sight-threatening or systemic conditions, possibly associated with mortality. When consultant ophthalmologists have ultimate responsibility for the patients seen in these clinics, they also take on these associated risks.

Whilst these risks can never be completely eliminated, consultant ophthalmologists who take responsibility for care delivered by other professionals need to consider what measures need to be put in place to minimise risk, and they must have confidence that the skills and knowledge of HCPs delivering care is to an appropriate level, given the degree of oversight possible by the consultant. Where possible HCPs should be encouraged and supported to obtain higher professional qualifications in order to practice autonomously and take full responsibility for the care they provide independently of the consultant, a matter considered separately below [5, 64].

\section{Training and accreditation for non-medical roles in the glaucoma care pathway}

Recommendations linking requirements for training and accreditation with specific roles in the glaucoma care pathway were first formally defined and published in the original NICE Glaucoma Guideline [30]. CG 85 defined three levels of extended 'permitted role' beyond contemporaneous roles in glaucoma care provision for ophthalmic non-medical HCPs and provided general descriptors of key training requirements for each. These roles, by ascending case complexity, were:

(i) Monitoring (but not treatment) of patients with OHT or suspected COAG with an established management plan.

(ii) Detection and diagnosis of $\mathrm{OHT}$ and glaucoma suspect status.

(iii) Monitoring and treatment of patients with OHT, suspected COAG and COAG.

The guideline listed role-specific clinical tests and assessments that HCPs should be trained in, both to perform and interpret, necessary to underpin clinical decisionmaking associated with the role. NICE recommendations explicitly stated that the roles be undertaken by trained HCPs, and that both specialist qualifications and experience were required for roles (ii) and (iii) when not working under consultant ophthalmologist supervision. The latter two roles were based largely upon content of the two levels of the CoO Glaucoma Higher Qualification (Certificates A and B) available at that time, although the language was used such that training and education were role specific and could be applied to any appropriate HCP.

The concept of the NICE-defined extended roles for nonmedical HCPs was developed further in the NICE-approved joint RCOphth and CCEHC Commissioning Guide: Glaucoma [6]. This document was designed "as a resource to assist commissioners, clinicians and managers to deliver high quality and evidence and outcome-based healthcare across England and beyond." The scope covered detection and diagnosis in addition to management, and therefore formed a valuable reference source for those involved in service design. In the context of monitoring, the roles defined in the NICE guideline, and by association their related training and accreditation requirements, were assimilated into the Commissioning Guide. The three NICE-defined roles originally were incorporated as levels I, III and IV of the Commissioning Guide. The Guide used the (then recently updated) $\mathrm{CoO}$ Glaucoma Higher Qualifications as an example of accreditation required for each role, with levels I, III and IV being equivalent to Optometric core competence, CoO Glaucoma Higher Professional Certificate 
and Professional Diploma respectively. An additional intermediate role, Level II, was added and recommended as a requirement for GERS, i.e. equivalent to the $\mathrm{CoO}$ Professional Certificate. The Commissioning Guide [6] stated that whilst $\mathrm{CoO}$ Higher Qualifications were used as an illustrative example, other qualifications which qualityassure the same NICE [5] CG85 levels of training would be equally acceptable.

In spite of the mapping of training and education and accreditation requirements with roles defined by both CG85 and the Commissioning Guide in 2015, access to training and accreditation remained limited to the three levels of $\mathrm{CoO}$ approved glaucoma higher qualifications. Publication of the OCCCF [64] in 2016 aimed to support development of training and accreditation for HCPs from different professional backgrounds in four key areas of eye care delivery including glaucoma. This framework was authored jointly by the RCOphth, RCN, CoO, British and Irish Orthoptic Society (BIOS) and the Association of Health Professions in Ophthalmology (AHPO) as a series of documents, setting out specific competences "that ophthalmic non-medical HCPs need to possess in order to safely and successfully undertake the expanded roles that they are currently performing". The OCCCF [64] was intended for use in development of education and training programmes with appropriate assessment processes. To this end, sub-specialty areas included in the OCCCF were developed into curricula [78], with supportive resources and assessment tools being made available online by Health Education England in 2019. The OCCCF defined three common levels of competence applied across the sub-specialties:

(1) Ability to participate in triage/screening and to monitoring low risk patients with an established diagnosis to a clearly defined clinical protocol.

(2) Ability to make preliminary diagnosis within a specific area and manage under specific protocols.

(3) Ability to diagnose, manage and discharge within specific areas of practice.

It is obvious that these three competence levels are matched and consistent with three roles described in both the NICE guideline [30] and Commissioning Guide [6]. For each of the named sub-specialties, the framework defined competences that must be achieved at each level in ophthalmic history taking, examination, investigations, management and interventions, ability to deal with needs of patients, teaching and education, personal development. Helpfully, in the context of glaucoma, OCCCF publications clearly acknowledge that these framework levels were mapped to recognise existing training programmes, giving again the example of the three levels of $\mathrm{CoO}$ Glaucoma Higher qualifications.
Final points to note in relation to training and accreditation requirements within the glaucoma care pathway are: The NICE glaucoma update [5], NG81, did not make changes to recommendations regarding roles and associated training and education requirements, but did remove the exception that NMPs working under supervision of a consultant ophthalmologist did not need to gain specialist accreditation relevant to their role; and furthermore, NG81 was careful to highlight the "holding an independent of nonmedical prescribing qualification alone (without a specialist qualification relevant to the case complexity of glaucoma being managed) is insufficient for managing glaucoma or related conditions.'

\section{Uncertainty on health economic issues in the care pathways}

There remains uncertainly about optimising care pathways within a wider health economics context. Burr et al.'s systematic review and economic evaluation of screening for open angle glaucoma [79] concluded population based screening was not justified in the UK and case finding continues as the primary pathway for detection. Interestingly in a recent editorial, Jonuscheit et al. [80] contrasted the GOS contract in Scotland with published evidence on primary community-based eye care services in England, albeit in a case comparison beyond that of glaucoma services alone. The authors argue that the Scottish GOS arrangements do provide value for money when compared with arrangements in England, concluding that the model provides enhanced ophthalmic services, free for all, permitting effective detection and management of acute eye conditions and improved quality of referrals to secondary care, albeit they concede knowledge gaps remain. As is the case for GRFSs discussed above, there is a paucity of published work detailing health economic evaluations of community monitoring of glaucoma. The realist review conducted by Baker et al. [3] noted that community glaucoma monitoring may be more expensive than if patients were monitored in the HES [81, 82], with factors contributing to higher community costs including equipment costs, shorter community monitoring intervals, high rates of re-referral back into the HES, and high opportunity costs to recover the lost income from the sale of spectacle. In terms of this latter point, the business model of community optometry is highly dependent on the cross-subsidy from spectacle sales in order to ensure profitability.

Setting aside the potential for debate about a hospital versus a community context for the care pathway, there are wider issues about sustainability of services and availability of resources to deliver care that some would argue might 
legitimately 'trump' economic matters for care providers; however, on an economic point, there is uncertainty about monitoring frequency, at least for OHT. Burr et al.'s evidence synthesis [83, 84] outlined that for confirmed OHT, monitoring more frequently than every 2 years was unlikely to be efficient and that while primary treatment and minimal monitoring (assessing IOP responsiveness to treatment) could be considered, further data via a cohort study was required on models for glaucoma risk prediction and patient preferences for treatment. In so far as COAG is concerned, Boodhna and Crabb's work [85] has highlighted the potential benefits of stratifying patients to more or less monitoring based upon age and disease staging at diagnosis, although they too concede that further prospective evaluation is necessary.

\section{Developments in future care pathways}

It is clear that the detection and monitoring of glaucoma presents an enormous challenge. Considerable strides have been made to: improve efficiency of detection; increase capacity for review appointments; and introduce improved standardisation of the quality of care within monitoring services (with strong collaboration across primary and secondary care and multidisciplinary working being vital to such progress); however, it is clear that further progress is needed. In terms of case finding, GRFS appear to work in terms of those seeking care but they do not reduce the burden of undetected disease or increase the equity of access to primary eye care. Developments in Scotland are noteworthy in this regard. In their paper arguing that aspects of the way the GOS Contract is implemented are contrary to the public health interest, Shickle et al. [86] suggest that the enhanced GOS model in Scotland should be judged by whether there is less of an incentive for community optometrists to cross-subsidise income through spectacle sales, thereby facilitating the opening of practices in more socioeconomically deprived areas. Time will tell, although one study examining practice distribution relative to deprivation has suggested that optometric practices in Scotland are relatively uniformly distributed across socioeconomic areas [87].

In terms of the clinical leadership of pathways for monitoring, there is as yet no guidance or consensus on the absolute numbers of glaucoma patients an individual consultant can reasonably take responsibility for. The Way Forward report [1] suggests that some units may have 6000-8000 patients per consultant, including non-medical HCP clinics, whilst in one region there was a solitary consultant covering a population of 80,000 , a scenario deemed to be unsustainable. This issue may be an area for future debate, as the demand for glaucoma services increase and consultants are faced with the concomitant greater burden of responsibility for patient care with which they have less immediate control over. With a predicted increase in glaucoma cases of $22 \%$ over the next 10 years, current models dependent upon consultant supervision require a significant increase in consultant numbers, and/or for nonmedical HCPs to take increasing responsibility for the care they deliver. At the same time, the role of artificial intelligence (AI), while showing promise, does not offer immediate solutions. In a recent review article on the use of AI in glaucoma, Zeng et al. [88] summarised that the techniques can successfully analyse and categorise data from measures of structure and function, ocular biomechanical properties, and a combination of these, in order to identify disease severity, determine disease progression, and/or recommend referral for specialised care. While it seems almost certain that the care pathways of the future will employ AI, possibly in a variety of scenarios, the potential for clinical and cost-effectiveness requires further research to better determine the ways in which such technology can be effectively implemented to improve glaucoma care pathways.

\section{Compliance with ethical standards}

Conflict of interest The authors declare that they have no conflict of interest.

Publisher's note Springer Nature remains neutral with regard to jurisdictional claims in published maps and institutional affiliations.

\section{References}

1. The Royal College of Ophthalmologists. The Way Forward: Options to help meet demand for the current and future care of patients with eye disease: Glaucoma. The Royal College of Ophthalmologists, London, January 2017.

2. Rutherford T. Population ageing: statistics. https://www.parlia mentuk/briefing-papers/sn03228pdf 2012; SN/SG/3228.

3. Baker H, Ratnarajan G, Harper RA, Edgar DF, Lawrenson JG. The effectiveness of enhanced optometric services in the management of acute and chronic ophthalmic disease: a realist review of the literature. Ophthalmic Physiol Opt. 2016;36:545-57.

4. https://www.nice.org.uk/guidance/qs180/chapter/Quality-sta tement-1-Referral-chronic-open-angle-glaucoma-and-relatedconditions.

5. https://www.nice.org.uk/guidance/ng81/evidence/full-guidelinepdf-4660991389.

6. Royal College of Ophthalmologists. Commissioning Guide: Glaucoma. Royal College of Ophthalmologists and the Clinical Council for Eye Health Commissioning, London, June 2016.

7. Gunn PG, Marks JR, Konstantakopoulou E, Edgar DF, Lawrenson JG, Roberts S, et al. Clinical effectiveness of the Manchester Glaucoma Enhanced Referral Scheme. Br J Ophthalmol. 2019;103:1066-71.

8. Henson DB, Spencer AF, Harper R, Cadman EJ. Community refinement of glaucoma referrals. Eye. 2003;17:21-6. 
9. Devarajan N, Williams GS, Hopes M, O’Sullivan D, Jones D. The Carmarthenshire Glaucoma Referral Refinement Scheme, a safe and efficient screening service. Eye. 2011;25:43-9.

10. Barrett C, O'Brien C, Loughman J. Glaucoma referral refinement in Ireland: managing the sensitivity-specificity paradox in optometric practice. Ophthal Physiol Opt. 2018;38:400-10. https://doi. org/10.1111/opo.12446. Epub 28 Feb 2018.

11. https://www.locsu.co.uk/commissioning/locsu-service-directory/ Accessed 24 June 2019.

12. Konstantakopoulou E, Harper RA, Edgar DF, Lawrenson JG. A qualitative study of stakeholder views regarding participation in locally commissioned enhanced optometric services. BMJ Open. 2014;4:e04781.

13. Baker H, Harper RA, Edgar DF, Lawrenson JG. Multi-stakeholder perspectives of locally commissioned enhanced optometric services. BMJ Open. 2016;6:e011934. https://doi.org/10.1136/bmjopen2016-011934.

14. Sheldrick JH, Ng C, Austin DJ, et al. An analysis of referral routes and diagnostic accuracy in cases of suspected glaucoma. Ophthalmic Epidemiol. 1994;1:31-9.

15. Bowling B, Chen SD, Salmon JF. Outcomes of referrals by community optometrists to a hospital glaucoma service. Br J Ophthalmol. 2005;89:1102-4.

16. Hernández R, et al. The clinical effectiveness and costeffectiveness of screening for open angle glaucoma: a systematic review and economic evaluation. Health Technol Assess. 2007;11:1-190.

17. Davey CJ, Green C, Elliott DB. Assessment of referrals to the hospital eye service by optometrists and GPs in Bradford and Airedale. Ophthalmic Physiol Opt. 2011;31:23-8.

18. Murdoch I, Theodossiades J. Is review of enriched populations the way forward for glaucoma case detection? Eye. 2003;17:5-6.

19. Founti P, Topouzis F, Holló G, Cvenkel B, Iester M, Haidich AB, et al. Prospective study of glaucoma referrals across Europe: are we using resources wisely? Br J Ophthalmol. 2018;102:329-37.

20. Harrison RJ, Wild JM, Hobley AJ. Referral patterns to an ophthalmic outpatient clinic by general practitioners and ophthalmic opticians and the role of these professionals in screening for ocular disease. BMJ. 1988;297:1162-7.

21. Tuck M, Crick RP. Efficiency of referral for suspected glaucoma. BMJ. 1991;302:998-1000.

22. Strong N. How optometrists screen for glaucoma: a survey. Ophthal Physiol Opt. 1992;12:3-7.

23. Clearkin L, Harcourt B. Referral pattern of true and suspected glaucoma to an adult ophthalmic outpatient clinic. Trans Ophthal Soc UK. 1983;103:284-7.

24. Bell RWD, OBrien C. The diagnostic outcome of new glaucoma referrals. Ophthal Physiol Opt. 1997;17:3-6.

25. Bell RWD, OBrien C. Accuracy of referral to a glaucoma clinic. Ophthal Physiol Opt. 1997;17:7-11.

26. Vernon SA. The changing pattern of glaucoma referrals by optometrists. Eye. 1998;12:854-7.

27. Theodossiades J, Murdoch L. Positive predictive value of optometrist-initiated referrals for glaucoma. Ophthal Physiol Opt. 1999;19:62-7.

28. Vernon SA, Ghosh G. Do locally agreed guidelines for optometrists concerning the referral of glaucoma suspects influence referral practice? Eye. 2001;15:458-63.

29. Ratnarajan G, Newsom W, Vernon SA, et al. The effectiveness of schemes that refine referrals between primary and secondary care - the UK experience with glaucoma referrals: the Health Innovation and Education Cluster (HIEC) Glaucoma Pathways Project. BMJ Open. 2013;3:e002715. https://doi.org/10.1136/ bmjopen-2013-002715.

30. NICE. Glaucoma: diagnosis and management, Clinical guideline [CG85], 2009. https://www.nice.org.uk/guidance/CG85.
31. Ratnarajan G, Kean J, French K, et al. The false negative rate and the role for virtual review in a nationally evaluated glaucoma referral refinement scheme. Ophthalmic Physiol Opt. 2015;35:577-81.

32. Ang GS, Ng WS, Azuara-Blanco A. The influence of the new general ophthalmic services (GOS) contract in optometrist referrals for glaucoma in Scotland. Eye. 2009;23:351-5.

33. El-Assal K, Foulds J, Dobson S, Sanders R. A comparative study of glaucoma referrals in Southeast Scotland: effect of the new general ophthalmic service contrast, Eyecare integration pilot programme and NICE guidelines. BMC Ophthalmol. 2015;15:1-8.

34. Scottish Intercollegiate Guidelines Network (SIGN). Glaucoma referral and safe discharge. Edinburgh: SIGN, 2015. Guideline No. 144.

35. Sii S, Nasser A, Loo CY, Croghan C, Rotchford A, Agarwal PK. The impact of SIGN glaucoma guidelines on false positive referrals from community optometrists in Central Scotland. Br J Ophthalmol. 2019;103:369-73.

36. Annoh R, Loo CY, Hogan B, Tan HL, Tang LS, Tatham AJ. Accuracy of detection of patients with narrow angles by community optometrists in Scotland. Ophthal Physiol Opt. 2019;39:104-12.

37. Black S, McClelland JF, Richardson P. An audit on the impact of training for a Referral Refinement Scheme in Northern Ireland on community optometrists' clinical practice when assessing for signs of glaucoma. Optom Pract. 2017;2017:27-40.

38. Parkins DJ, Edgar DF. Comparison of the effectiveness of two enhanced glaucoma referral schemes. Ophthalmic Physiol Opt. 2011;31:343-52.

39. Ratnarajan G, Newsom W, French K, et al. The impact of glaucoma referral refinement criteria on referral to, and first-visit discharge rates from, the hospital eye service: the Health Innovation \& Education Cluster (HIEC) Glaucoma Pathways project. Ophthalmic Physiol Opt. 2013;33:183-9.

40. Trikha S, Macgregor C, Jeffery M, Kirwan J. The Portsmouthbased glaucoma refinement scheme: a role for virtual clinics in the future? Eye. 2012;26:1288-94.

41. Sarkies N. Costs of shared care. Eye. 2005;19:475.

42. Manners T. Reference: Community refinement of glaucoma referrals. Eye. 2005;19:475.

43. Forbes H, Sutton M, Edgar DF, Lawrenson J, Spencer AF, Fenerty C, et al. Impact of the Manchester Glaucoma Enhanced Referral scheme on NHS costs. BMJ Open Ophthalmol. 2019;4: e000278.

44. Rathod D, Win T, Pickering S, et al. Incorporation of a virtual assessment into a care pathway for initial glaucoma management: feasibility study. Clin Exp Ophthalmol. 2008;36:543-6.

45. Azuara-Blanco A, Banister K, Boachie C, et al. Automated imaging technologies for the diagnosis of glaucoma: a comparative diagnostic study for the evaluation of the diagnostic accuracy, performance as triage tests and cost effectiveness (GATE study). Health Technol Assess. 2016;20:1-168.

46. Hitchings R. Shared care for glaucoma. $\mathrm{Br} \mathrm{J}$ Ophthalmol. 1995;79:626.

47. Vernon SA, Adair A. Shared Care in glaucoma: a national study of secondary care lead schemes in England. Eye. 2010;24:265-9.

48. Harper R, Creer R, Jackson A, Ehrlich DP, Tompkins A, Bowen $\mathrm{M}$, et al. Scope of practice of hospital optometrists in the UK: a national survey. Ophthalmic Physiol Opt. 2016;36:197-206.

49. Gray SF, Spencer IC, Spry PG, et al. The Bristol Shared Care Glaucoma Study - validity of measurements and patient satisfaction. J Public Health Med. 1997;19:431-6.

50. Spry PG, Spencer IC, Sparrow JM, et al. The Bristol Shared Care Glaucoma Study: reliability of community optometric and hospital eye service test measures. Br J Ophthalmol. 1999;83:707-12.

51. Gray SF, Spry PG, Brookes ST, et al. The Bristol shared care glaucoma study: outcome at follow up at 2 years. Br J Ophthalmol. 2000;84:456-63. 
52. Azuara-Blanco A, Burr J, Thomas R, Maclennan G, McPherson S. The accuracy of accredited glaucoma optometrists in the diagnosis and treatment recommendation for glaucoma. $\mathrm{Br} \mathrm{J}$ Ophthalmol. 2007;91:1639-43.

53. Banes MJ, Culham LE, Bunce C, Xing W, Viswanathan A, Garway-Heath D. Agreement between optometrists and ophthalmologists on clinical management decisions for patients with glaucoma. Br J Ophthalmol. 2006;90:579-85.

54. Ho S, Vernon SA. Decision making in chronic glaucomaoptometrists vs ophthalmologists in a shared care service. Ophthalmic Physiol Opt. 2011;31:168-73.

55. Chawla A, Patel I, Yuen C, Fenerty C. Patterns of adherence to NICE glaucoma guidance in two different service delivery models. Eye. 2012;26:1412-7.

56. Marks JR, Harding AK, Harper RA, et al. Agreement between specially trained and accredited optometrists and glaucoma specialist consultant ophthalmologists in their management of glaucoma patients. Eye. 2012;26:853-61.

57. Syam P, Rughani K, Vardy SJ, et al. The Peterborough scheme for community specialist optometrists in glaucoma: a feasibility study. Eye. 2010;24:1156-64.

58. Mandalos A, Bourne R, French K, Newsom W, Chang L. Shared care of patients with ocular hypertension in the Community and Hospital Allied Network Glaucoma Evaluation Scheme (CHANGES). Eye. 2012;26:564-7.

59. Roberts HW, Rughani K, Syam P, Dhingra S, Ramirez- Florez S. The Peterborough scheme for community specialist optometrists in glaucoma: results of 4 years of a two-tiered community-based assessment and follow-up service. Curr Eye Res. 2014;13:1-7.

60. Ney JJ. Glaucoma diagnosis and treatment: the role of the ophthalmic nurse. Insight. 2016;41:13-7.

61. Johnson ZK, Griffiths PG, Birch MK. Nurse prescribing in glaucoma. Eye. 2003;17:47-52.

62. Gibbons H, Bourne RR. Extending a nurse practitioner's role to include the undertaking of advanced procedures. Nurs. 2009; 105:24-6

63. Kotecha A, Elkarmouty A, Ajtony C, Barton K. Interobserver agreement using Goldmann applanation tonometry and dynamic contour tonometry: comparing ophthalmologists, nurses and technicians. Br J Ophthalmol. 2016;100:854-9.

64. The Common Clinical Competency Framework for Non-medical Ophthalmic Healthcare Professionals in Secondary Care: Glaucoma. RCOphth, November 2016.

65. Ophthalmic Services Guidance: Primary Eye Care, Community Ophthalmology and General Ophthalmology. Royal College of Ophthalmologists and College of Optometrists, February 2019. https://www.rcophth.ac.uk/wp-content/uploads/2019/02/PrimaryEye-Care-Community-Ophthalmology-and-General-Ophtha lmology-2019.pdf.

66. The College of Optometrists (2018). How UK eye care services are delivered. The College of Optometrists. London. https://collegeoptometrists.org/the-college/how-uk-eye-care-services-are-delivered.

67. Slanke A, Poustie M. Developing eye care partnerships 2012-27. Improving the commissioning and provision of eye care services in Northern Ireland. Department of Health. P1-58.

68. Together for Health: Eye Health Care - Delivery Plan for Wales 2013-2018. Welsh Government. P1-33.

69. Clinical Council For Eye Health Commissioning. System and assurance framework for eye-health (SAFE)—Glaucoma, March 2018.
70. Wright HR, Diamond JP. Service innovation in glaucoma management: using a web-based electronic patient record to facilitate virtual specialist supervision of a shared care glaucoma programme. Br J Ophthalmol. 2015;99:313-7.

71. Kotecha A, Baldwin A, Brookes J, et al. Experiences with developing and implementing a virtual clinic for glaucoma care in an NHS setting. Clin Ophthalmol. 2015;9:1915-23.

72. Gunn PJG, Marks JR, Au L, Waterman H, PGDS Spry, Harper RA. Acceptability and use of glaucoma virtual clinics in the UK: a national survey of clinical leads. BMJ Open Ophthalmol. 2018;3: e000127. https://doi.org/10.1136/bmjophth-2017-000127.

73. Royal College of Ophthalmologists. Standards for virtual clinics in glaucoma care in the NHS Hospital Eye Service. 2016. https://www.rcophth.ac.uk/wp-content/uploads/2017/03/VirtualGlaucoma-Clinics.Pdf.

74. Clarke J, Puertas R, Kotecha A, et al. Virtual clinics in glaucoma care: face-to-face versus remote decision-making. Br J Ophthalmol. 2017;101:892-5.

75. Kotecha A, Bonstein K, Cable R, et al. Qualitative investigation of patients' experience of a glaucoma virtual clinic in a specialist ophthalmic hospital in London, UK. BMJ Open. 2015;5:e009463.

76. Court JH, Austin MW. Virtual glaucoma clinics: patient acceptance and quality of patient education compared to standard clinics. Clin Ophthalmol. 2015;9:745-9.

77. Broadway DC, Tibbenham K. Tackling the NHS glaucoma clinic backlog issue. Eye. 2019;33:1715-21.

78. OCCCF online curricula, 2019. https://www.hee.nhs.uk/our-w ork/advanced-clinical-practice/ophthalmology-common-clinicalcompetency-framework-curriculum.

79. Burr JM, Mowatt G, Hernández R, et al. The clinical effectiveness and cost-effectiveness of screening for open angle glaucoma: a systematic review and economic evaluation. Health Technol Assess. 2007;11:1-190. iii-iv, ix-x.

80. Jonuscheit S, Loffler G, Strang NC. General ophthalmic services in Scotland: value for (public) money? Ophthal Physiol Opt. 2019;39:225-31.

81. Coast J, Spencer IC, Smith L, Spry PG. Comparing costs of monitoring glaucoma patients: hospital ophthalmologists versus community optometrists. J Health Serv Res Policy. 1997;2:19-25.

82. Sharma A, Jofre-Bonet M, Panca M, Lawrenson JG, Murdoch I. An economic comparison of hospital-based and community-based glaucoma clinics. Eye. 2012;26:967-71.

83. Hernandez R, Burr JM, Vale L, et al. Monitoring ocular hypertension, how much and how often? A cost-effectiveness perspective. Br J Ophthalmol. 2016;100:1263-8.

84. Burr JM, Botello-Pinzon P, Takwoingi Y, et al. Surveillance for ocular hypertension: an evidence synthesis and economic evaluation. Health Technol Assess. 2012;16:1-271. iii-iv.

85. Boodhna T, Crabb DP. More frequent, more costly? Health economic modelling aspects of monitoring glaucoma patients in England. BMC Health Serv Res. 2016;16:611.

86. Shickle D, Davey CJ, Slade SV. Why is the General Ophthalmic Services (GOS) contract that underpins primary eye care in the UK contrary to the public health interest? $\mathrm{Br} \mathrm{J}$ Ophthalmol. 2015;99:888-92.

87. Legge R, Strang NC, Loffler G. Distribution of optometric practices relative to deprivation index in Scotland. J Public Health. 2018;40:389-96.

88. Zheng C, Johnson TV, Garg A, Boland MV. Artificial intelligence in glaucoma. Curr Opin Ophthalmol. 2019;30:97-103. 